Mon. Not. R. Astron. Soc. 000, 1 10(2015) Printed 7 February $2018 \quad$ (MN LATEX style file v2.2)

\title{
Characterizing long-term optical, ultraviolet and X-ray variability in different activity states of OJ 287
}

\author{
H. Siejkowski ${ }^{1 \star}$, A. Wierzcholska ${ }^{\dagger} \dagger$, \\ ${ }^{1}$ AGH University of Science and Technology, ACC Cyfronet AGH, ul. Nawojki 11, PO Box 386, PL-30-950, Kraków 23, Poland \\ ${ }^{2}$ Institute of Nuclear Physics, Polish Academy of Sciences, ul. Radzikowskiego 152, PL-31-342 Kraków, Poland
}

Accepted .... Received ...; in original form ...

\begin{abstract}
We have studied long-term optical, ultraviolet (UV) and X-ray observations of OJ 287 collected with the UVOT and XRT instruments mounted on board the Swift satellite to quantify spectral and temporal variability patterns observed during different activity states. We characterized the flux variations using the data collected during almost $11 \mathrm{yr}$ of the monitoring of the blazar. Significant variability of the blazar has been detected both in the flux and spectral index from the optical to X-ray regimes. We noted that the variability patterns observed in the optical range are more pronounced than the ones in the X-ray band. There is no clear relation between the optical/UV and X-ray emission, neither during the quiescence state nor during outbursts. The most significant flares in the optical/UV regime were detected in 2015 December2016 January. The shortest variability time-scale is one day and it is limited by the observation pointing. A low activity state of OJ 287 was observed at the end of 2014, while the beginning of 2015 revealed a flat X-ray spectrum, which has been observed for the first time. On one hand, this can be a spectral upturn where the synchrotron and inverse Compton components meet, but on the other hand, it can be generated by an additional emission component. The spectral studies have not revealed any bluerwhen-brighter or redder-when-brighter chromatism in the colour-magnitude diagram for OJ 287 in any state of the source's activity. A harder-when-brighter behaviour was noticed for OJ 287 only in the case of the X-ray observations.
\end{abstract}

Key words: radiation mechanisms: non-thermal, galaxies: active, BL Lacertae objects: general, BL Lacertae objects: individual: OJ 287

\section{INTRODUCTION}

Blazars, including BL Lacertae (BL Lac) type objects and flat spectrum radio quasars (FSRQs), represent a violent class of active galactic nuclei (AGNs). These are sources whose jets are pointing at a small angle with respect to the observer's line of sight (e.g. Begelman et al. 1984). Blazars are known to be highly variable with variability observed at different time-scales from minutes to years (e.g. Wagner \& Witzel 1995, Aharonian et al. 2007 Gopal-Krishna et al. 2011; Saito et al. 2013; Wierzcholska \& Siejkowski|2015a). The observed emission extends from radio frequencies up to a high and very high energy regime (e.g. Wagner 2009 H.E.S.S. Collaboration 2014, Wierzcholska 2015). The spectral energy distribution (SED) of blazars, in $\nu-\nu F_{\nu}$ representation, exhibits a double-humped structure. The first, low-

* E-mail: h.siejkowski@cyfronet.pl

† E-mail: alicja.wierzcholska@ifj.edu.pl energy bump is usually attributed to the synchrotron emission of relativistic electrons from the jet, while the second is still a matter of debate, and leptonic, hadronic and hybrid scenarios are applied (see e.g. Maraschi et al. 1992, Sikora et al. 1994; Kirk et al. 1998, Mücke et al. 2003; Böttcher et al. 2013).

The two classes of blazars: BL Lac objects and FSRQs can be distinguished by different features in optical/UV spectra (Urry \& Padovani 1995). In the case of BL Lac type sources, featureless continuum emission is observed, while spectra of FSRQs are characterized with broad and narrow emission lines. BL Lac type objects can be further subdivided into high-, intermediate- and low-energy-peaked BL Lac type objects (HBL, IBL, LBL, respectively) and this classification is based upon the position of the low-energy peak in the SED (see, e.g., Padovani \& Giommi 1995, Fossati et al. 1998, Abdo et al. 2010). Following Abdo et al. (2010), for LBL sources the low-energy peak is located in the regime defined as $\nu_{\mathrm{s}} \leqslant 10^{14} \mathrm{~Hz}$ (infrared range), for 
IBL ones this regime is $10^{14}<\nu_{\mathrm{s}} \leqslant 10^{15} \mathrm{~Hz}$ (optical/UV range), while in the case of $\mathrm{HBL}$ blazars $\nu_{\mathrm{s}}>10^{15} \mathrm{~Hz}$ (X-ray range). HBLs-IBLs-LBLs-FSRQs constitute a blazar sequence. This connection of decreasing bolometric luminosities and $\gamma$-ray dominance has been proposed and discussed by Fossati et al. (1998) and Ghisellini et al. (1998) and has been updated into blazars envelope by Meyer et al. (2011). Furthermore, Ghisellini et al. (2011) studied the properties of SEDs and emission lines of a large sample of sources from a one-year all-sky survey by the Fermi satellite and proposed a physical distinction between FSRQs and BL Lac objects based on the luminosity of the broad line region.

OJ 287 ( $z=0.306)$ has become one of the best monitored blazars in the optical regime since its identification in this range by Dickel et al. (1967). The source is classified as an LBL type object. Deeper studies of the source started in the 1980 s and therefore OJ 287 is a perfect candidate for long-term variability studies and searching for periodicities. An almost 12-yr periodicity is observed in the optical monitoring of OJ 287 with two peaks observed during every flaring event (e.g. Valtonen et al. 2006). Additionally, a 60yr variability was claimed by Valtonen et al. (2006). This behaviour is usually explained in terms of the binary black hole model (see e.g. Sillanpaa et al. 1988, 1996). An indication for a shorter periodic behaviour in the optical range was suggested by Sagar et al. (2004), Wu et al. (2006), Gupta et al. (2012), and Valtonen \& Wiik (2012).

Despite many years of observations, OJ 287 has not been well studied in the other energy regimes except for the optical and radio ones. This work presents $12 \mathrm{yr}$ of multifrequency observations of OJ 287 preformed with the XRT and UVOT instruments onboard the Swift space telescope. The paper is organized as follows: Section 2 presents the data analysis details. Section 3 and 4 focus on a characterization of the temporal and spectral variability of OJ 287, respectively. The work is summarized in Section 5

\section{DATA ANALYSIS}

\section{$2.1 \quad$ X-ray observations}

X-ray observations made with the XRT telescope on board the Swift satellite (see Gehrels et al. 2004 for details) in the energy range of $0.3-10 \mathrm{keV}$ were analysed using version 6.19 of the HEASOFT package. In these studies, all observations made with Swift/XRT in the PC mode from the mission start up to MJD 57552.93053 are considered. The total number of observations analysed is 280 . All the data were reprocessed using the standard XRTPIPELINE procedur£ ${ }^{1}$ In the case of each observation for the spectral fitting, XSPEC (v.12.9.0n) was used (Arnaud 1996). All light-curve points were derived by fitting the power-law model with the value of the Galactic absorption frozen at $N_{\mathrm{H}}=2.56 \times 10^{20} \mathrm{~cm}^{-2}$ taken from Kalberla et al. (2005). The observations were corrected for the pile-up in the $\mathrm{PC}$ mode whenever the count rate was 0.5 or higher.

\subsection{Optical and ultraviolet observations}

Simultaneously with the XRT instrument, OJ 287 was monitored with Swift/UVOT in the optical/UV band. The optical and UV observations were taken in six bands: $U V W 2$ (188 nm), UVM2 (217 nm), UVW1 (251 nm), U (345 nm), B $(439 \mathrm{~nm})$ and $V(544 \mathrm{~nm})$. The uvotsource procedure was used in order to calculate the instrumental magnitudes in the aperture with a radius of 5 arcsec. The background area was defined as a circular region with a radius of $5 \operatorname{arcsec}$ located close to the source region and not being contaminated with any signal from the nearby sources. The influence of three different sizes of background was checked and the results obtained were consistent within the uncertainties. All magnitudes were converted into fluxes using the conversion factors provided by Poole et al. (2008). The data were corrected for dust contamination using the reddening $E(B-V)=0.0241$ from Schlafly \& Finkbeiner (2011) and ratios of the extinction to reddening ratios from Giommi et al. (2006).

\section{TEMPORAL VARIABILITY STUDIES}

During the period of 2005-2016, OJ 287 was observed many times with Swift/UVOT and Swift/XRT resulting in 280 pointing observations. The long-term optical, UV and X-ray light curve of the source is presented in Fig. 1. The upper panel of the plot shows the optical observations performed with Swift/UVOT in the $U, B$, and $V$ filters; the middle one shows the UV observations made in $U V W 1, U V M 2$ and $U V W 2$ filters; and the bottom one shows the X-ray monitoring performed with Swift/XRT in the energy range of $2-10 \mathrm{keV}$. The energy range chosen allowed us to compare the results obtained in previous works focusing on an X-ray data analysis. For the temporal variability studies, we used the entire set of data as well as nine intervals marked in Fig. 1 with vertical grey areas and letters A-I. Each interval represents a similar flux level and consists of series of consecutive observations.

Strong flux changes were observed in the X-ray data as well as in the optical and UV range. A comparison of the flux light curves for the data collected in the X-ray as well as in the optical and UV regimes does not show any clear relation between the different energy ranges. Strong optical/UV outbursts did not have so strong counterparts in the $\mathrm{X}$-ray regime and inversely. The discrete correlation function (DCF, Edelson \& Krolik 1988) does not reveal any relation between the optical/UV and X-ray observations (Fig. 2). In order to obtain the DCF, we used the algorithm implemented by Alexander (1997).

Let us notice here that OJ 287 is a LBL type blazar and in the case of such a source the optical/UV range corresponds to the first, low-energy bump in the SED, while the high energy one is placed in the X-ray regime. This causes that the changes observed in the optical/UV regime and the X-ray one may be caused by different physical processes responsible for the emission observed.

Fig. 3 shows a comparison of the X-ray and optical $V$ band fluxes. The result presented in the plot confirms no relation visible in the light-curve plot (see Fig. 1). Pearson's correlation coefficient for this case is $C=-0.005$, which 


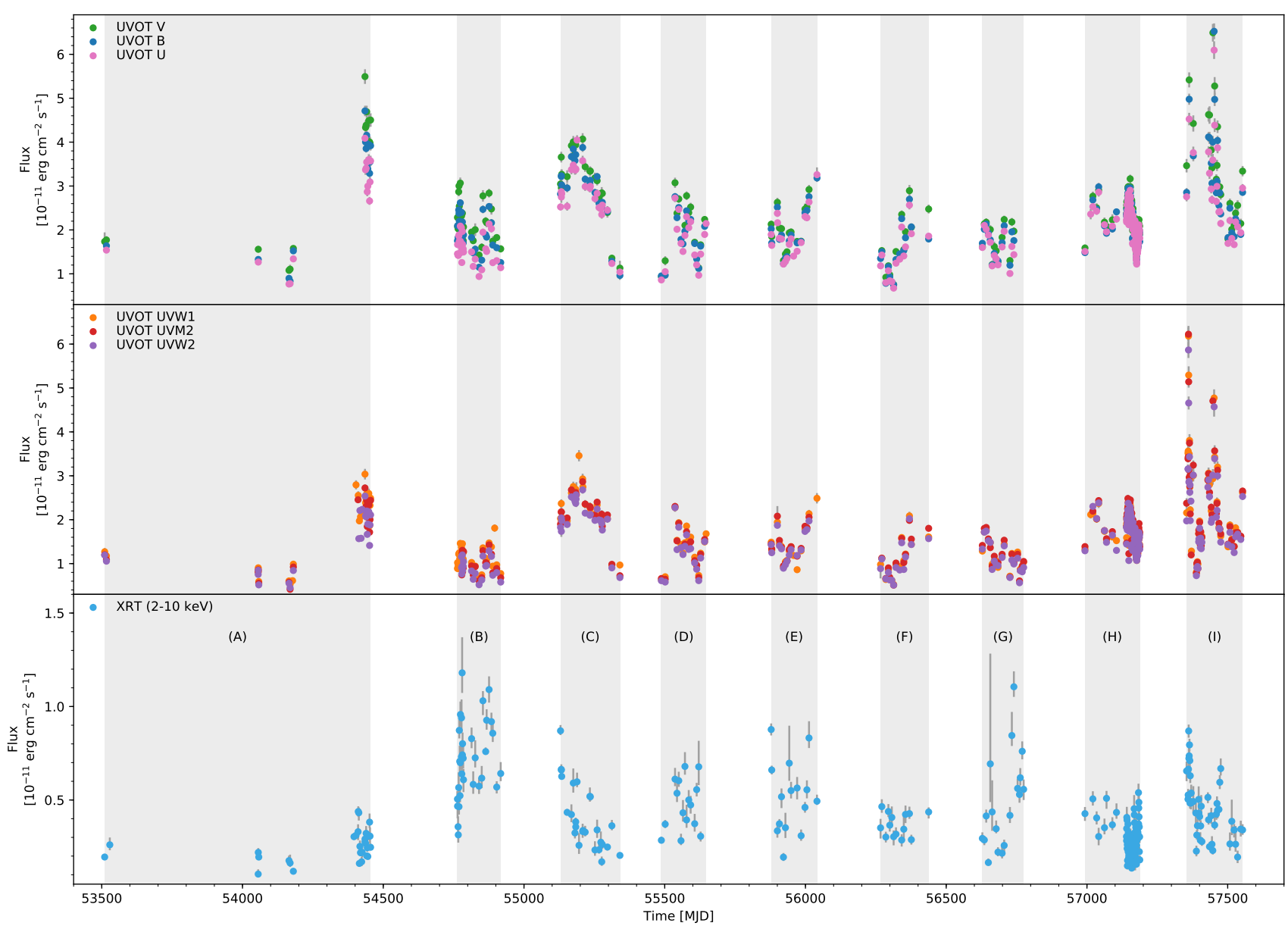

Figure 1. The long-term light curve presenting the optical, UV and X-ray observations of OJ 287 made during the period of $2005-2016$. The vertical grey areas indicate the A-I intervals chosen for detailed studies.

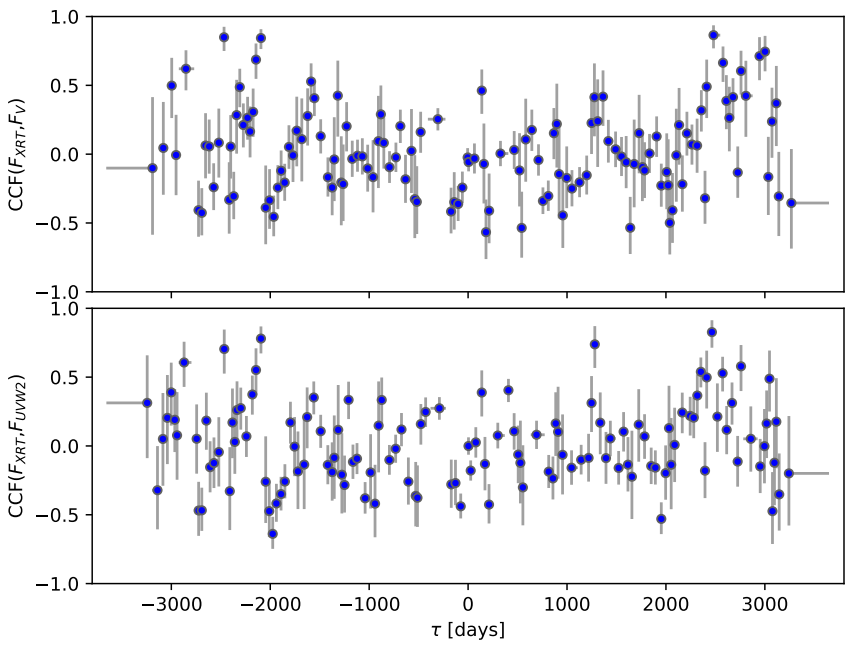

Figure 2. The DCF between the X-ray flux $\left(F_{\mathrm{XRT}}\right)$ and the filter $V$ flux $\left(F_{V}\right)$ and filter $U V W 2$ flux $\left(F_{U V W 2}\right)$.

indicates no clear correlation between these two ranges. The same result, a lack of any clear relation, has been obtained for the comparison of the X-ray band with every optical/UV band.



Figure 3. A comparison of the X-ray and optical fluxes collected in the $V$ band.

As a first step of a characterization of the UV, optical and X-ray long-term variability of the blazar, the fractional variability amplitude was used. It is defined by Vaughan et al. (2003a) as follows: 


$$
F_{\text {var }}=\sqrt{\frac{S^{2}-e r r^{2}}{F^{2}}},
$$

where $F$ is the mean flux and $S^{2}$ is its variance and $e r r^{2}$ is the mean square error. The errors of the fractional variability amplitude are calculated following the formula by Poutanen et al. (2008):

$\delta F_{\mathrm{var}}=\sqrt{F_{\mathrm{var}}^{2}+\left(\sigma^{2}\right)}-F_{\mathrm{var}}$,

where the error in the normalized excess variance $\sigma$ is calculated following Vaughan et al. (2003b):

$\sigma=\sqrt{\left(\sqrt{\frac{2}{N}} \frac{e r r^{2}}{F^{2}}\right)^{2}+\left(\sqrt{\frac{e r r^{2}}{N}} \frac{2 F_{\mathrm{var}}}{F}\right)^{2}}$,

where $N$ indicates the number of data points in the light curve.

In the next step, the doubling/halving time-scale was used in order to characterize the variability of OJ 287 . The quantity is defined in two ways (following e.g. Zhang et al. 1999):

(i) as the smallest value of the set constituted of $(k, m)$ pairs:

$\tau_{k, m}=\left|\frac{\Phi \Delta T}{\Delta \Phi}\right|=\left|\frac{\left(\Phi_{k}+\Phi_{m}\right)\left(T_{k}-T_{m}\right)}{2\left(\Phi_{k}-\Phi_{m}\right)}\right|$ and

(ii) the mean value of the five smallest items from $\left\{\tau_{k, m}\right\}$.

The flux value at the time of $T_{j}$ is denoted by $\Phi_{j}$, for all pairs of points $(k, m)$ in the light curve. The doubling/halving time-scale for the definition (i) is marked as $\tau_{\mathrm{d}}^{(i)}$, while for (ii) as $\tau_{\mathrm{d}}^{(i i)}$. The latter value can be used as a cross check for the $\tau_{\mathrm{d}}^{(i)}$. Single minimum value can be generated by chance, and the $\tau_{\mathrm{d}}^{(i i)}$ gives information how small is the minimum compared to the five smallest values (mainly due to irregular sampling). If the $\tau_{\mathrm{d}}^{(i)}$ is much different from the $\tau_{d}^{(i i)}$ then the probability that the minimum is accidental is high.

The variability parameters are collected in Table 1 and 2 for the X-ray and optical/UV observations, respectively. In addition to the fractional variability amplitude and doubling time-scale, the tables present the mean flux value and the reduced $\chi^{2}$ of the fit with a constant value. All quantities were calculated for all the data collected during the period between 2005 and 2016 and for the nine separate intervals (A-I).

The fractional variability amplitude and $\chi^{2}$ value confirm significant variability observed in all the energy ranges studied. The most prominent variability for all optical and UV filters is observed for interval A. The fractional variability amplitude for this interval is between 0.44 and 0.49 . In the case of the X-ray monitoring, the largest value of $F_{\text {var }}$ is for the entire set of data. The doubling time-scale values calculated determine the shortest variability time-scale observed in the long-term monitoring of OJ 287 as one day.

\section{SPECTRAL VARIABILITY STUDIES}

For the optical/UV observations in order to derive the spectral index of emission in this band, we fitted a power law to the data gathered with Swift/UVOT for each ObsID.



Figure 4. The time evolution of the long-term spectral indices for the optical/UV range (top panel) and for the X-ray band (bottom panel). The optical/UV spectral index is found by fitting a power law to data points (for details see Sect. 4).

We only used observations that have measurements in each filter for a given ObsID (i.e. six data points). The fitting was done with the optimize.curve_fit function from the SCIPY version 0.18.0 package (Jones et al. 2001), which uses the Levenberg-Marquardt algorithm. Fig. 4 shows the evolution in time of the X-ray and optical/UV spectral indices, while Fig. 5 presents a plot of the X-ray versus optical/UV spectral index. The spectral index in the case of the X-ray observations was obtained with a power-law fit to a single observation.

In the case of the X-ray observations, the spectral index varies between 1.19 and 2.43, while for the optical/UV ones, the range is $2.03-2.89$. The plots show significant variability in the spectral index's evolution for all wavebands. The changes observed both in the spectral index's evolution and the correlation plots show a strong anti-correlation between these two quantities. The correlation coefficient for this comparison is $C=-0.71 \pm 0.04$. The uncertainty of Pearson's correlation coefficient was estimated using a Monte Carlo approach following Wierzcholska (2015).

We also compare the X-ray spectral index with the corresponding flux (see Fig. 6). The correlation visible in the plot, characterized with Pearson's correlation coefficient of $C=-0.67 \pm 0.02$, indicates a harder-when-brighter behaviour in the long-term observations. This spectral hardening with the increasing flux is a typical feature of HBL type sources (see e.g. Pian et al. 1998, Zhang et al. 2005).

We have also noted a lack of correlation between the fluxes observed in the X-ray and optical/UV regimes. This is in agreement with the absence of a harder-when-brighter relation in the flux-index diagram for the optical observations (see Fig. 7).

\subsection{Colour-magnitude diagram}

Three colour-magnitude diagrams, namely $(B-V)$ versus $B,(B-U)$ versus $B,(V-U)$ versus $V$, are presented in Fig. 8 For each case, the Pearson's correlation coefficients were found to be equal to $0.05,0.11$ and 0.13 for the corre- 


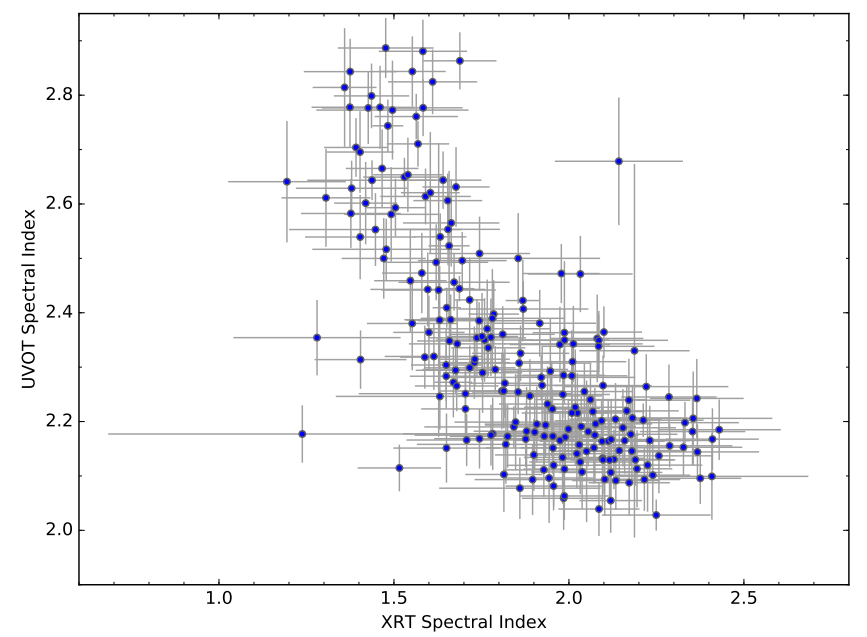

Figure 5. A comparison of the spectral indices obtained from X-ray and optical/UV observations.

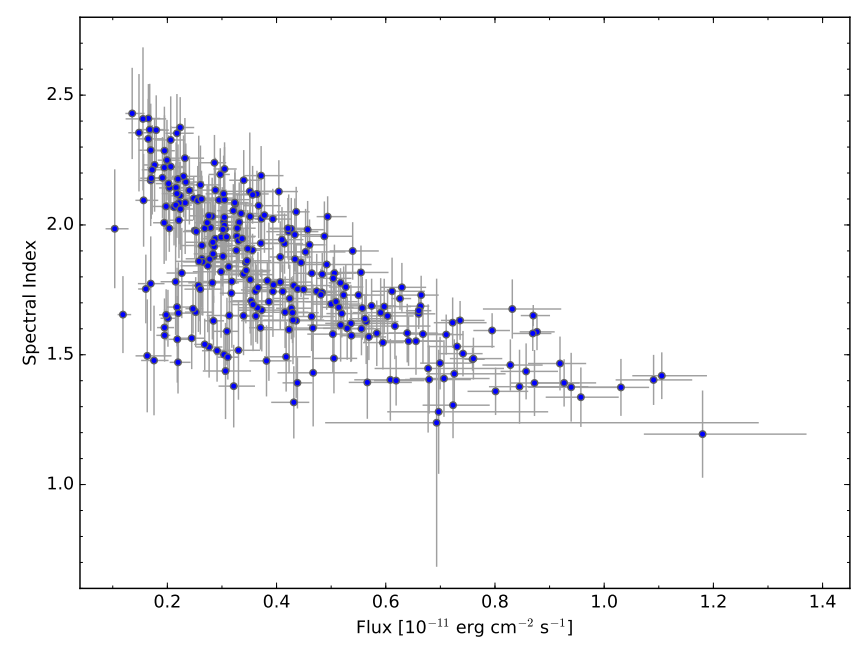

Figure 6. A comparison of the X-ray flux and the corresponding spectral index obtained from the power-law fit.

sponding colour-magnitude diagrams, respectively. The correlation coefficients found suggest that there is no significant bluer-when-brighter or redder-when-brighter trend in the entire set of the observations discussed. Furthermore, the analysis of shorter intervals of observations both in the low and high states also did not show any chromatism observed in the Swift/UVOT observations.

Previous works focusing on the colour-magnitude relation in OJ 287 have revealed various types of behaviours. Some hints for a bluer-when-brighter chromatism were reported by Carini et al. (1992) while studying observations collected in the $V$ and $B$ bands in 1973-1976. A clear bluerwhen-brighter chromatism during a flaring state was found by Dai et al. (2011). The observations mentioned have been collected in the $R$ and $V$ bands from 1993 to 1997. Similarly, Ikejiri et al. (2011) found an indication for a weak colour-magnitude correlation in the observations collected from 2008 May to 2010 January in the $V$ and $J$ filters. A lack of a bluer-when-brighter or redder-when-brighter relation was noticed for shorter time-scales, as well as for long-
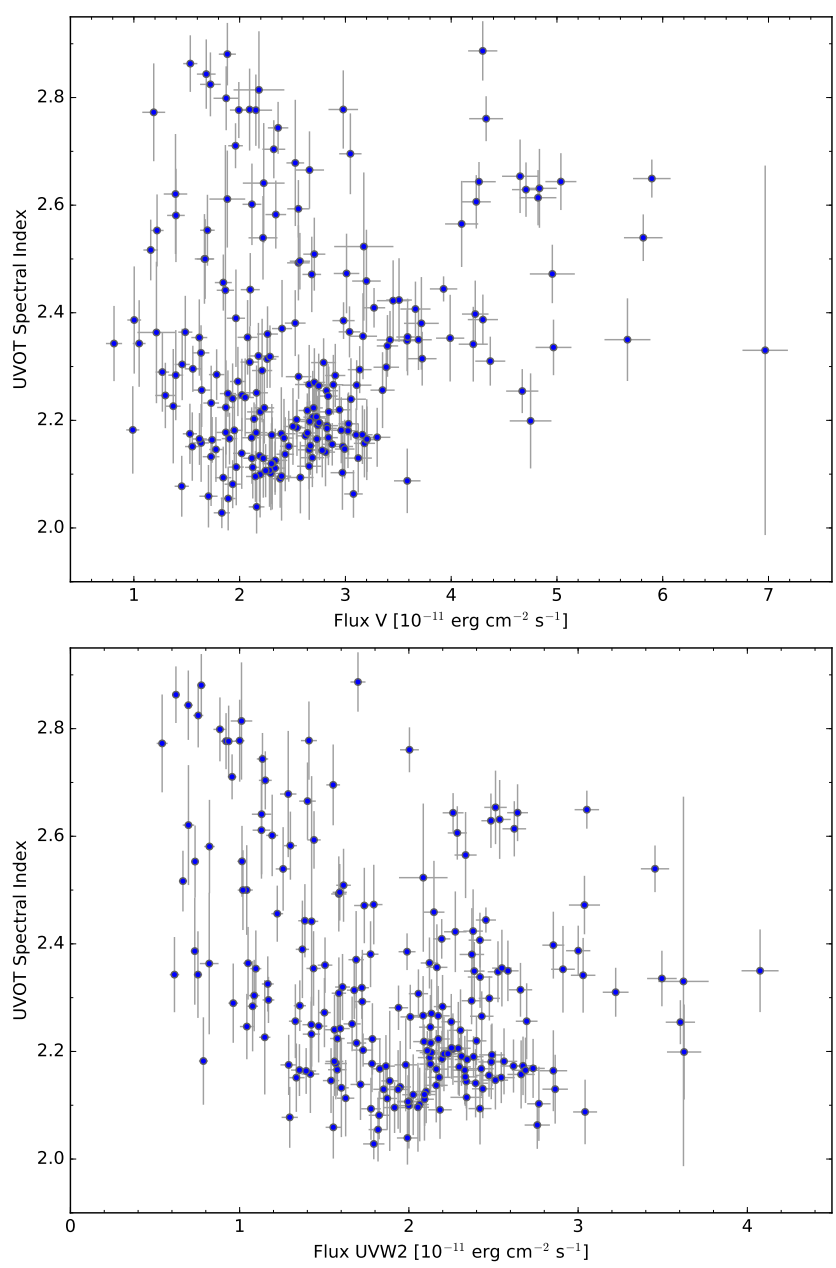

Figure 7. A comparison of the optical flux in the $V$ filter (top panel) and the optical/UV spectral index obtained from the power-law fit (see Sect. 4). The bottom panel shows an analogical relation but for the $U V W 2$ filter.

term observations collected in the $B$ and $R$ bands during the period 2007-2012 with the ATOM telescope (Wierzcholska et al. 2015).

The various behaviour of the optical colour as a function of the magnitude observed of OJ 287 indicates a complex behaviour of the optical emission mechanisms at work in this blazar. An absence of a bluer-when-brighter chromatism can be caused by multiple episodes characterized with different bluer-when-brighter slopes. In such a case, the relation cannot be visible in a large set of observations, but can be detected in shorter periods.

\subsection{Optical-UV-X-ray SEDs}

Fig. 9 shows the SED for the nine intervals defined in Sect. 3. The parameters of the X-ray spectra are listed in Table 3. All of the nine cases show that the soft optical/UV spectrum corresponds to the hard X-ray one, and vice versa. Moreover, the following pattern emerges: The harder the optical/UV spectra, the softer the X-ray spectra. We also note that in the case of the $\mathrm{H}$ interval, the X-ray spectrum is characterized with a spectral index of about 2.0. We consider two possible scenarios explaining such an evolution of the X-ray 

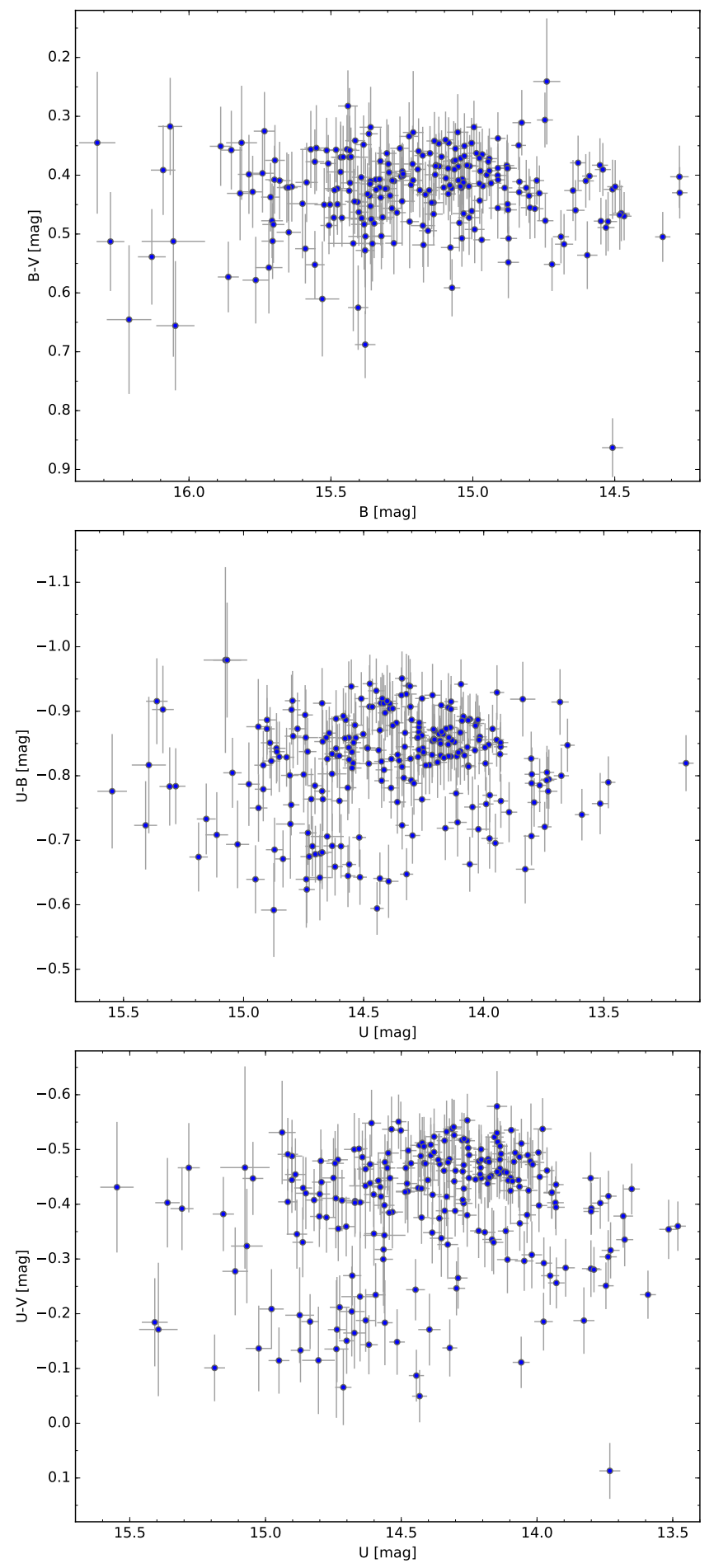

Figure 8. Colour-magnitude diagrams for the optical observations performed with Swift/UVOT. The plots present a comparison of: $(B-V)$ versus $B,(U-B)$ versus $U,(U-V)$ versus $U$.

spectrum. On one hand, this spectral shape can be caused by the fact that the X-ray regime is a place where two spectral components meet and the flat spectrum is a consequence of an overlap of these spectral components. Such a feature is known for many LBL type blazars (see e.g. Wierzcholska \& Siejkowski 2016, Wierzcholska \& Wagner 2016), but has never been reported for OJ 287 before. On the other hand, this flat spectrum can be an effect of an additional spectral component, which can be detected only in the case of the low state of OJ 287. This can be, for example the Bethe-Heitler emission as proposed by Petropoulou \& Mastichiadis (2015). In such a case, the synchrotron emission from Bethe-Heitler pairs is expected to appear as a third bump - an additional component with a maximum at tens of $\mathrm{keV}$ - in blazar's SED. Its signature can be seen as a lack of a spectral upturn in a broad-band SED.

\section{SUMMARY}

OJ 287 has been observed by astronomers for many years, but most of the observations focused mainly on the optical regime (e.g. Takalo et al. 1994; Pian et al. 1995; Sillanpaa et al. 1996, Pursimo et al. 2000, Valtonen et al. 2016). This paper focuses on detailed studies of the spectral and temporal variability from optical to $\mathrm{X}$ rays of this famous LBL blazar. The long-term observations made with Swift/UVOT and Swift/XRT during the period of 2005-2016 are discussed. This work can be summarized as follows:

- Significant variability observed in the optical, UV and $\mathrm{X}$-ray ranges has been confirmed in the long-term observations of OJ 287. The description of the blazar's behaviour revealed the strongest flux changes observed in the optical/UV regime during the flare observed in 2015 December and 2016 January. However, in the X-ray regime the simultaneous outburst is not so pronounced. An extraordinary activity of OJ 287 over the entire electromagnetic spectrum during the period mentioned has been reported (see e.g. Carrasco et al. 2015, Ciprini et al. 2015. Shappee et al. 2015. Wierzcholska \& Siejkowski 2015b).

- The shortest variability time-scale observed is one day and this is strongly limited by the pointing of the observations.

- The variability patterns observed in the optical/UV and $\mathrm{X}$-ray ranges are not correlated.

- The long-term optical monitoring of the source has not revealed any clear relation between colour and magnitude. Such a trend has been previously reported but mostly in the case of short-term observations in the high states of the blazar (e.g. Carini et al. 1992; Dai et al. 2011).

- A harder-when-brighter behaviour is clearly visible in the case of the X-ray data, but not in the case of the optical/UV band.

- A statistically significant and strong anti-correlation between the optical/UV and X-ray spectral indices has been found.

- In the case of the low state of the blazar observed in 2014-2015 (here interval H), a flat X-ray spectrum has been observed. This feature can be explained as a spectral upturn observed in the X-ray regime or this may be an effect of the influence of an additional spectral component, like the Bethe-Heitler emission, observed in the low state.

\section{ACKNOWLEDGEMENTS}

A.W. acknowledges support by the Foundation for Polish Science (FNP). This research was supported in part by 




Figure 9. SED for OJ 287 for the intervals defined in Sect. 3 The colour points represent the optical/UV observations and the grey points - the X-ray observations. The butterfly plots show the power-law model with the uncertainty of the fit at a confidence level of $1 \sigma$.

PLGrid Infrastructure. The plots presented in this paper are rendered using Matplotlib (Hunter 2007).

\section{REFERENCES}

Abdo A. A. et al., 2010, ApJ, 716, 30

Aharonian F. et al., 2007, ApJ, 664, L71
Alexander T., 1997, in Astrophysics and Space Science Library, Vol. 218, Astronomical Time Series, Maoz D., Sternberg A., Leibowitz E. M., eds., p. 163

Arnaud K. A., 1996, in Astronomical Society of the Pacific Conference Series, Vol. 101, Astronomical Data Analysis Software and Systems V, Jacoby G. H., Barnes J., eds., p. 17

Begelman M. C., Blandford R. D., Rees M. J., 1984, Reviews of Modern Physics, 56, 255 
Table 1. A characterization of the variability of OJ 287 using the X-ray observations. The following columns show: the instrument/filter, the interval, time period in MJD, the mean flux value given in $10^{-11} \mathrm{erg} \mathrm{cm}^{-2} \mathrm{~s}^{-1}$, the reduced chi squares $\chi_{\text {red }}^{2}$ value of the fit to a constant value and the number of degrees of freedom, the fractional variability amplitude, and two values of the doubling/halving time-scale, as defined in Section 3 given in days. The intervals are defined in Section 3 and additionally, we calculate the quantities for the whole data set - marked as interval 'all'.

\begin{tabular}{lcccrrrr}
\hline \hline Instrument & \multirow{2}{*}{ Interval } & Period & Mean value & $\chi_{\text {red }}^{2}$ & \multicolumn{1}{c}{$F_{\text {var }}$} & \multicolumn{1}{c}{$\tau_{\mathrm{d}}^{(i)}$} & \multicolumn{1}{c}{$\tau_{\mathrm{d}}^{(i i)}$} \\
\hline XRT & $\mathrm{A}$ & $53510.27-54455.02$ & $0.25 \pm 0.02$ & $12.0 / 28$ & $0.311 \pm 0.022$ & $<0.01$ & 1.61 \\
$\mathrm{XRT}$ & $\mathrm{B}$ & $54763.67-54917.26$ & $0.72 \pm 0.04$ & $10.9 / 30$ & $0.274 \pm 0.018$ & 1.77 & 1.88 \\
$\mathrm{XRT}$ & $\mathrm{C}$ & $55129.78-55340.81$ & $0.41 \pm 0.03$ & $36.0 / 24$ & $0.409 \pm 0.019$ & 1.91 & 6.24 \\
$\mathrm{XRT}$ & $\mathrm{D}$ & $55488.02-55647.06$ & $0.47 \pm 0.04$ & $14.9 / 14$ & $0.256 \pm 0.035$ & 8.94 & 12.64 \\
$\mathrm{XRT}$ & $\mathrm{E}$ & $55878.18-56040.85$ & $0.52 \pm 0.05$ & $38.3 / 13$ & $0.358 \pm 0.037$ & 6.89 & 12.80 \\
$\mathrm{XRT}$ & $\mathrm{F}$ & $56266.74-56437.64$ & $0.36 \pm 0.02$ & $3.0 / 12$ & $0.119 \pm 0.038$ & 16.05 & 21.40 \\
$\mathrm{XRT}$ & $\mathrm{G}$ & $56628.38-56775.11$ & $0.49 \pm 0.07$ & $40.0 / 15$ & $0.417 \pm 0.085$ & 5.59 & 10.70 \\
$\mathrm{XRT}$ & $\mathrm{H}$ & $56992.94-57187.06$ & $0.29 \pm 0.01$ & $7.3 / 81$ & $0.289 \pm 0.016$ & 0.30 & 0.40 \\
XRT & $\mathrm{I}$ & $57187.61-57552.93$ & $0.45 \pm 0.02$ & $21.9 / 45$ & $0.357 \pm 0.016$ & 2.10 & 2.96 \\
XRT & all & $53510.27-57552.93$ & $0.41 \pm 0.01$ & $31.9 / 279$ & $0.473 \pm 0.009$ & $<0.01$ & 0.29 \\
\hline \hline
\end{tabular}

Böttcher M., Reimer A., Sweeney K., Prakash A., 2013, ApJ, 768, 54

Carini M. T., Miller H. R., Noble J. C., Goodrich B. D., 1992, AJ, 104, 15

Carrasco L., Recillas E., Porras A., Chavushyan V., Leon-

Tavares J., Carraminana A., 2015, The Astronomer's Telegram, 8438

Ciprini S., Perri M., Verrecchia F., Valtonen M., 2015, The Astronomer's Telegram, 8401

Dai Y., Wu J., Zhu Z.-H., Zhou X., Ma J., 2011, AJ, 141, 65

Dickel J. R., Yang K. S., McVittie G. C., Swenson, Jr. G. W., 1967, AJ, 72, 757

Edelson R. A., Krolik J. H., 1988, ApJ, 333, 646

Fossati G., Maraschi L., Celotti A., Comastri A., Ghisellini G., 1998, MNRAS, 299, 433

Gehrels N. et al., 2004, ApJ, 611, 1005

Ghisellini G., Celotti A., Fossati G., Maraschi L., Comastri A., 1998, MNRAS, 301, 451

Ghisellini G., Tavecchio F., Foschini L., Ghirlanda G., 2011, MNRAS, 414, 2674

Giommi P. et al., 2006, A\&A, 456, 911

Gopal-Krishna, Goyal A., Joshi S., Karthick C., Sagar R., Wiita P. J., Anupama G. C., Sahu D. K., 2011, MNRAS, 416, 101

Gupta S. P., Pandey U. S., Singh K., Rani B., Pan J., Fan J. H., Gupta A. C., 2012, New A, 17, 8

H.E.S.S. Collaboration, 2014, A\&A, 571, A39

Hunter J. D., 2007, Computing In Science \& Engineering, 9,90

Ikejiri Y. et al., 2011, PASJ, 63, 639

Jones E., Oliphant T., Peterson P., et al., 2001, SciPy: Open source scientific tools for Python. [Online; accessed 2016-08-23]

Kalberla P. M. W., Burton W. B., Hartmann D., Arnal E. M., Bajaja E., Morras R., Pöppel W. G. L., 2005, A\&A, 440, 775

Kirk J. G., Rieger F. M., Mastichiadis A., 1998, A\&A, 333, 452

Maraschi L., Ghisellini G., Celotti A., 1992, ApJ, 397, L5

Meyer E. T., Fossati G., Georganopoulos M., Lister M. L., 2011, ApJ, 740, 98
Mücke A., Protheroe R. J., Engel R., Rachen J. P., Stanev T., 2003, Astroparticle Physics, 18, 593

Padovani P., Giommi P., 1995, ApJ, 444, 567

Petropoulou M., Mastichiadis A., 2015, MNRAS, 447, 36

Pian E. et al., 1995, Advances in Space Research, 16, 57

Pian E. et al., 1998, ApJ, 492, L17

Poole T. S. et al., 2008, MNRAS, 383, 627

Poutanen J., Zdziarski A. A., Ibragimov A., 2008, MNRAS, 389, 1427

Pursimo T. et al., 2000, A\&AS, 146, 141

Sagar R., Stalin C. S., Gopal-Krishna, Wiita P. J., 2004, MNRAS, 348, 176

Saito S., Stawarz Ł., Tanaka Y. T., Takahashi T., Madejski G., D'Ammando F., 2013, ApJ, 766, L11

Schlafly E. F., Finkbeiner D. P., 2011, ApJ, 737, 103

Shappee B. J. et al., 2015, The Astronomer's Telegram, 8372

Sikora M., Begelman M. C., Rees M. J., 1994, ApJ, 421, 153

Sillanpaa A., Haarala S., Valtonen M. J., Sundelius B., Byrd G. G., 1988, ApJ, 325, 628

Sillanpaa A. et al., 1996, A\&A, 315, L13

Takalo L. O., Sillanpaeae A., Nilsson K., 1994, A\&AS, 107

Urry C. M., Padovani P., 1995, PASP, 107, 803

Valtonen M. J. et al., 2006, ApJ, 646, 36

Valtonen M. J., Wiik K., 2012, MNRAS, 421, 1861

Valtonen M. J. et al., 2016, ApJ, 819, L37

Vaughan S., Edelson R., Warwick R. S., Uttley P., 2003a, MNRAS, 345, 1271

Vaughan S., Edelson R., Warwick R. S., Uttley P., 2003b, MNRAS, 345, 1271

Wagner S., 2009, in Astrophysics with All-Sky X-Ray Observations, Kawai N., Mihara T., Kohama M., Suzuki M., eds., p. 186

Wagner S. J., Witzel A., 1995, ARA\&A, 33, 163

Wierzcholska A., 2015, A\&A, 580, A104

Wierzcholska A., Ostrowski M., Stawarz Ł., Wagner S., Hauser M., 2015, A\&A, 573, A69

Wierzcholska A., Siejkowski H., 2015a, MNRAS, 452, L11

Wierzcholska A., Siejkowski H., 2015b, The Astronomer's Telegram, 8395

Wierzcholska A., Siejkowski H., 2016, MNRAS, 458, 2350 
Table 2. A characterization of the variability of OJ 287 using the optical and UV observations. The meaning of the following columns is the same as in Table 1 .

\begin{tabular}{|c|c|c|c|c|c|c|}
\hline Instrument & Interval & Mean value & $\chi_{\text {red }}^{2}$ & $F_{\text {var }}$ & $\tau_{\mathrm{d}}^{(i)}$ & $\tau_{\mathrm{d}}^{(i i)}$ \\
\hline UVOT V & A & $3.21 \pm 0.39$ & $259.3 / 14$ & $0.474 \pm 0.011$ & 8.51 & 17.58 \\
\hline UVOT V & B & $2.20 \pm 0.08$ & $19.6 / 28$ & $0.181 \pm 0.011$ & 2.80 & 3.78 \\
\hline UVOT V & $\mathrm{C}$ & $3.19 \pm 0.14$ & $53.4 / 20$ & $0.194 \pm 0.009$ & 1.18 & 14.17 \\
\hline UVOT V & $\mathrm{D}$ & $2.02 \pm 0.18$ & $78.2 / 13$ & $0.328 \pm 0.011$ & 18.01 & 26.38 \\
\hline UVOT V & $\mathrm{E}$ & $2.01 \pm 0.12$ & $30.5 / 14$ & $0.224 \pm 0.011$ & 13.64 & 24.49 \\
\hline UVOT V & $\mathrm{F}$ & $1.61 \pm 0.20$ & $66.8 / 10$ & $0.406 \pm 0.015$ & 12.59 & 20.66 \\
\hline UVOT V & $\mathrm{G}$ & $1.79 \pm 0.10$ & $19.8 / 13$ & $0.197 \pm 0.013$ & 13.24 & 21.16 \\
\hline UVOT V & $\mathrm{H}$ & $2.27 \pm 0.05$ & $32.5 / 85$ & $0.188 \pm 0.004$ & 2.59 & 2.84 \\
\hline UVOT V & I & $3.42 \pm 0.26$ & $95.6 / 22$ & $0.368 \pm 0.008$ & 5.26 & 9.94 \\
\hline UVOT V & all & $2.43 \pm 0.06$ & $76.5 / 233$ & $0.364 \pm 0.003$ & 1.18 & 2.46 \\
\hline UVOT B & $\mathrm{A}$ & $2.90 \pm 0.36$ & $511.1 / 13$ & $0.462 \pm 0.008$ & 10.36 & 15.53 \\
\hline UVOT B & $\mathrm{B}$ & $1.93 \pm 0.07$ & $40.2 / 30$ & $0.185 \pm 0.007$ & 1.85 & 2.42 \\
\hline UVOT B & $\mathrm{C}$ & $3.02 \pm 0.13$ & $91.1 / 20$ & $0.194 \pm 0.006$ & 19.36 & 22.36 \\
\hline UVOT B & $\mathrm{D}$ & $1.85 \pm 0.14$ & $115.3 / 15$ & $0.298 \pm 0.008$ & 18.26 & 26.94 \\
\hline UVOT B & $\mathrm{E}$ & $1.90 \pm 0.12$ & $58.5 / 14$ & $0.233 \pm 0.008$ & 11.25 & 23.19 \\
\hline UVOT B & $\mathrm{F}$ & $1.46 \pm 0.16$ & $125.5 / 13$ & $0.399 \pm 0.010$ & 15.11 & 19.13 \\
\hline UVOT B & $\mathrm{G}$ & $1.68 \pm 0.09$ & $34.6 / 13$ & $0.194 \pm 0.009$ & 14.06 & 26.68 \\
\hline UVOT B & $\mathrm{H}$ & $2.16 \pm 0.05$ & $70.3 / 84$ & $0.201 \pm 0.003$ & 2.14 & 2.71 \\
\hline UVOT B & I & $3.16 \pm 0.25$ & $161.2 / 23$ & $0.383 \pm 0.007$ & 7.13 & 9.09 \\
\hline UVOT B & all & $2.25 \pm 0.05$ & $146.6 / 240$ & $0.367 \pm 0.002$ & 1.85 & 2.26 \\
\hline UVOT U & $\mathrm{A}$ & $2.52 \pm 0.30$ & $432.3 / 13$ & $0.451 \pm 0.010$ & 9.54 & 11.75 \\
\hline UVOT U & $\mathrm{B}$ & $1.59 \pm 0.05$ & $33.8 / 30$ & $0.187 \pm 0.007$ & 1.87 & 2.71 \\
\hline UVOT U & $\mathrm{C}$ & $2.88 \pm 0.12$ & $73.4 / 22$ & $0.194 \pm 0.007$ & 5.91 & 19.87 \\
\hline UVOT U & $\mathrm{D}$ & $1.73 \pm 0.14$ & $115.9 / 15$ & $0.318 \pm 0.009$ & 17.01 & 24.19 \\
\hline UVOT U & $\mathrm{E}$ & $1.82 \pm 0.10$ & $44.9 / 16$ & $0.225 \pm 0.009$ & 7.65 & 20.02 \\
\hline UVOT U & $\mathrm{F}$ & $1.35 \pm 0.14$ & $106.1 / 13$ & $0.397 \pm 0.011$ & 14.10 & 18.79 \\
\hline UVOT U & $\mathrm{G}$ & $1.58 \pm 0.09$ & $34.7 / 13$ & $0.209 \pm 0.010$ & 14.52 & 23.79 \\
\hline UVOT U & $\mathrm{H}$ & $2.12 \pm 0.04$ & $52.9 / 86$ & $0.193 \pm 0.003$ & 2.11 & 2.43 \\
\hline UVOT U & $\mathrm{I}$ & $2.90 \pm 0.22$ & $113.9 / 23$ & $0.373 \pm 0.008$ & 5.89 & 8.56 \\
\hline UVOT U & all & $2.11 \pm 0.05$ & $129.7 / 247$ & $0.359 \pm 0.002$ & 1.87 & 2.17 \\
\hline UVOT UVW1 & $\mathrm{A}$ & $1.76 \pm 0.17$ & $338.8 / 22$ & $0.464 \pm 0.009$ & 3.68 & 5.38 \\
\hline UVOT UVW1 & $\mathrm{B}$ & $1.12 \pm 0.04$ & $24.8 / 32$ & $0.210 \pm 0.008$ & 1.87 & 2.47 \\
\hline UVOT UVW1 & $\mathrm{C}$ & $2.30 \pm 0.10$ & $51.1 / 23$ & $0.199 \pm 0.009$ & 1.42 & 9.99 \\
\hline UVOT UVW1 & $\mathrm{D}$ & $1.36 \pm 0.11$ & $97.7 / 15$ & $0.335 \pm 0.011$ & 13.10 & 22.12 \\
\hline UVOT UVW1 & $\mathrm{E}$ & $1.40 \pm 0.09$ & $40.0 / 15$ & $0.258 \pm 0.011$ & 14.74 & 23.61 \\
\hline UVOT UVW1 & $\mathrm{F}$ & $1.05 \pm 0.11$ & $75.9 / 13$ & $0.406 \pm 0.013$ & 13.37 & 16.42 \\
\hline UVOT UVW1 & G & $1.20 \pm 0.07$ & $31.6 / 16$ & $0.248 \pm 0.011$ & 12.76 & 21.89 \\
\hline UVOT UVW1 & $\mathrm{H}$ & $1.77 \pm 0.04$ & $25.9 / 86$ & $0.189 \pm 0.005$ & 0.80 & 1.66 \\
\hline UVOT UVW1 & $\mathrm{I}$ & $2.35 \pm 0.17$ & $192.6 / 44$ & $0.487 \pm 0.007$ & 1.99 & 3.14 \\
\hline UVOT UVW1 & all & $1.71 \pm 0.04$ & $129.9 / 283$ & $0.430 \pm 0.003$ & 0.80 & 1.44 \\
\hline UVOT UVM2 & $\mathrm{A}$ & $1.59 \pm 0.18$ & $422.3 / 17$ & $0.487 \pm 0.008$ & 4.03 & 6.37 \\
\hline UVOT UVM2 & $\mathrm{B}$ & $0.99 \pm 0.06$ & $33.1 / 17$ & $0.241 \pm 0.015$ & 1.31 & 2.73 \\
\hline UVOT UVM2 & $\mathrm{C}$ & $2.22 \pm 0.08$ & $65.8 / 21$ & $0.170 \pm 0.009$ & 14.72 & 19.11 \\
\hline UVOT UVM2 & $\mathrm{D}$ & $1.33 \pm 0.13$ & $151.4 / 13$ & $0.368 \pm 0.010$ & 12.02 & 20.82 \\
\hline UVOT UVM2 & $\mathrm{E}$ & $1.45 \pm 0.09$ & $43.3 / 14$ & $0.242 \pm 0.014$ & 18.88 & 25.29 \\
\hline UVOT UVM2 & $\mathrm{F}$ & $1.11 \pm 0.13$ & $129.8 / 10$ & $0.399 \pm 0.012$ & 12.64 & 20.78 \\
\hline UVOT UVM2 & $\mathrm{G}$ & $1.19 \pm 0.08$ & $58.1 / 17$ & $0.289 \pm 0.009$ & 8.27 & 14.14 \\
\hline UVOT UVM2 & $\mathrm{H}$ & $1.80 \pm 0.04$ & $34.4 / 85$ & $0.194 \pm 0.004$ & 0.63 & 1.15 \\
\hline UVOT UVM2 & $\mathrm{I}$ & $2.33 \pm 0.17$ & $319.0 / 44$ & $0.487 \pm 0.005$ & 1.51 & 2.17 \\
\hline UVOT UVM2 & all & $1.73 \pm 0.05$ & $185.9 / 253$ & $0.428 \pm 0.003$ & 0.63 & 1.05 \\
\hline UVOT UVW2 & $\mathrm{A}$ & $1.51 \pm 0.14$ & $399.5 / 20$ & $0.436 \pm 0.008$ & 2.90 & 4.83 \\
\hline UVOT UVW2 & B & $0.91 \pm 0.05$ & $47.2 / 19$ & $0.227 \pm 0.010$ & 1.74 & 3.11 \\
\hline UVOT UVW2 & $\mathrm{C}$ & $2.09 \pm 0.08$ & $72.0 / 22$ & $0.176 \pm 0.007$ & 1.57 & 9.80 \\
\hline UVOT UVW2 & $\mathrm{D}$ & $1.25 \pm 0.11$ & $170.1 / 15$ & $0.364 \pm 0.009$ & 11.42 & 17.95 \\
\hline UVOT UVW2 & $\mathrm{E}$ & $1.38 \pm 0.08$ & $49.8 / 14$ & $0.233 \pm 0.009$ & 14.11 & 23.96 \\
\hline UVOT UVW2 & $\mathrm{F}$ & $0.99 \pm 0.11$ & $102.5 / 13$ & $0.411 \pm 0.019$ & 13.18 & 16.51 \\
\hline UVOT UVW2 & $\mathrm{G}$ & $1.13 \pm 0.08$ & $84.1 / 17$ & $0.299 \pm 0.009$ & 7.00 & 13.88 \\
\hline UVOT UVW2 & $\mathrm{H}$ & $1.70 \pm 0.04$ & $31.7 / 87$ & $0.194 \pm 0.004$ & 0.50 & 1.09 \\
\hline UVOT UVW2 & I & $2.22 \pm 0.16$ & $261.9 / 45$ & $0.472 \pm 0.006$ & 1.54 & 2.13 \\
\hline UVOT UVW2 & all & $1.62 \pm 0.04$ & $211.7 / 267$ & $0.429 \pm 0.002$ & 0.50 & 1.05 \\
\hline
\end{tabular}


Table 3. The parameters of the power-law spectrum in the Xray regime for a given interval. The power-law is defined as: $N(E / 1 \mathrm{keV})^{-\gamma}$, where $N$ is the normalization, $E$ is the energy and $\gamma$ is the index.

\begin{tabular}{rcc}
\hline \hline Interval & $N\left(10^{-3} \mathrm{~cm}^{-2} \mathrm{~s}^{-1} \mathrm{keV}^{-1}\right)$ & $\gamma$ \\
\hline $\mathrm{A}$ & $0.476 \pm 0.008$ & $1.585 \pm 0.020$ \\
$\mathrm{~B}$ & $1.222 \pm 0.017$ & $1.495 \pm 0.016$ \\
$\mathrm{C}$ & $1.369 \pm 0.014$ & $1.811 \pm 0.014$ \\
$\mathrm{D}$ & $0.997 \pm 0.020$ & $1.707 \pm 0.026$ \\
$\mathrm{E}$ & $1.406 \pm 0.024$ & $1.793 \pm 0.023$ \\
$\mathrm{~F}$ & $1.088 \pm 0.026$ & $1.763 \pm 0.032$ \\
$\mathrm{G}$ & $1.087 \pm 0.021$ & $1.705 \pm 0.026$ \\
$\mathrm{H}$ & $1.215 \pm 0.010$ & $2.072 \pm 0.013$ \\
$\mathrm{I}$ & $1.220 \pm 0.013$ & $1.755 \pm 0.014$ \\
\hline \hline
\end{tabular}

Wierzcholska A., Wagner S. J., 2016, MNRAS, 458, 56

Wu J. et al., 2006, AJ, 132, 1256

Zhang Y. H. et al., 1999, ApJ, 527, 719

Zhang Y. H., Treves A., Celotti A., Qin Y. P., Bai J. M., 2005, ApJ, 629, 686 